\title{
Los museos veterinarios: puntos de encuentro entre la historia y la ciencia
}

\author{
Veterinary Museums: Meeting Points between History \\ and Science \\ Naudy Trujillo Mascia \\ Universidad Centroccidental Lisandro Alvarado (UCLA), Venezuela \\ naudytrujillo@ucla.edu.ve
}

\section{Resumen}

Este ESCAPARATE es una contribución informativa que expone aspectos del movimiento de los museos veterinarios con el objeto de hacer aportes al conjunto de conocimientos de la museística.

\section{Palabras clave \\ museos; historia; veterinaria}

\section{Abstract}

This GALLERY is an informative contribution that explains some aspects of the veterinary museum movement with the aim of making contributions to the knowledge of museum studies.

\section{Keywords}

museums; history; veterinary science<smiles>B</smiles>
ara una buena cantidad de lectores resultará muy interesante saber que cada una de las facultades y muchas asociaciones de medicina veterinaria del mundo poseen al menos un estante con alguna colección de objetos que ilustra la trayectoria de la profesión; más aún, que incluso hay las que han establecido grandes museos, a cuyo resguardo se tiene una variedad de piezas, archivos o artefactos con innegable valor pedagógico, histórico y de promoción de la identidad y la memoria colectiva de esta disciplina científica y su entorno social (Figura 1).

De tal manera, estos entes museísticos de veterinaria pretenden conservar sus propias colecciones, grandes o pequeñas, en las que incluyen una amplia gama de categorías: piezas anatómicas (Figura 2 y 3), patológicas y parasitológicas; artefactos de carácter histórico; instrumentos, herramientas y aparatos obsoletos; documentos y libros; indumentarias, uniformes y símbolos, e incluso objetos de bellas artes relacionados con las ciencias veterinarias, la ganadería (Figura 4) o la cultura de mascotas.

De hecho, esta tradición en términos de conservación, que no sólo cumple con el tricotómico papel de cualquier iniciativa museística de preservar, in- 


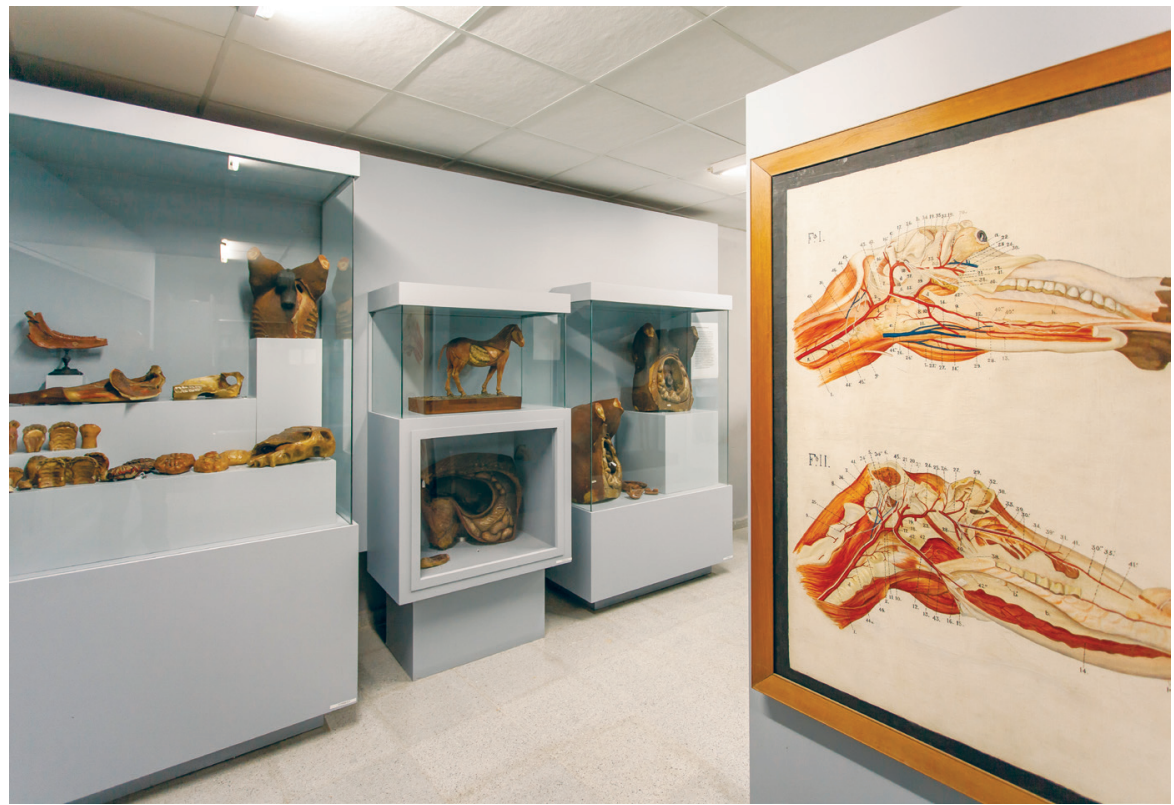

FIGURA 1. Vista de la sala del almacén abierto al público del Museo Veterinario Complutense, en el Hospital Clínico Veterinario, mostrando óleos de anatomía y parte de la colección de piezas de cera policromada realizadas en el primer tercio del siglo XIX. Patrimonio Histórico Universidad Complutense de Madrid (Cortesía: Museo Veterinario Complutense [MVC], Universidad Complutense de Madrid [UCM]).

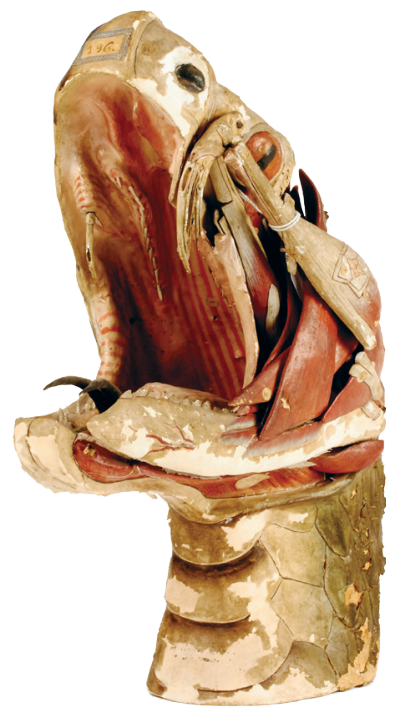

FIGURA 2. Modelo anatómico de cabeza de serpiente de Louis Thomas Jerôme Auzoux, papel maché, depósito del Departamento de Anatomía y Anatomía Patológica Comparada (Anatomía y Embriología). Patrimonio Histórico Universidad Complutense de Madrid (Cortesía: Museo Veterinario Complutense [MVC], Universidad Complutense de Madrid [UCM]).

vestigar y educar, sino también sirve como puente comunicativo entre el hombre y su pasado, la globalización de la cultura y la tecnología, que es antiguo en la medicina veterinaria. Se inició en la misma época en que

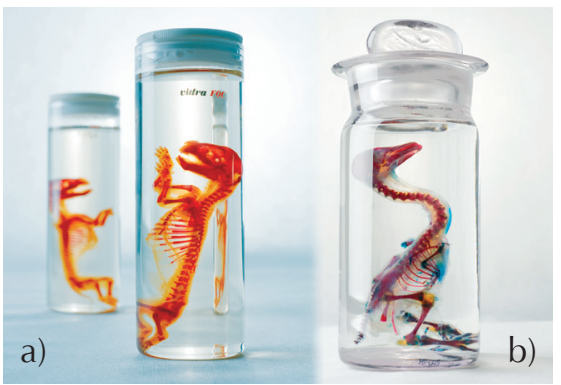

FIGURA 3. Transparentizaciones a) rata; b) pato, preparación en fluido, glicerina, depósito del Departamento de Anatomía y Anatomía Patológica Comparada (Anatomía y Embriología). Patrimonio Histórico Universidad Complutense de Madrid (Cortesía: Museo Veterinario Complutense [MVC], Universidad Complutense de Madrid [UCM]).

surgieron sus escuelas superiores en el mundo, a partir de 1761, con la creación de la Ecole Vétérinaire de Lyon, Francia, por Claude Bourgelat (1712-1779). Así, en la segunda institución de este tipo, creada en 1763, la Ecole Vétérinaire de Alfort, cerca de París, se estableció tres años después el museo Honoré Fragonard, que aún está en funcionamiento (Degueurce 2012:16-17).

Recuérdese que, a raíz de las grandes exploraciones y descubrimientos

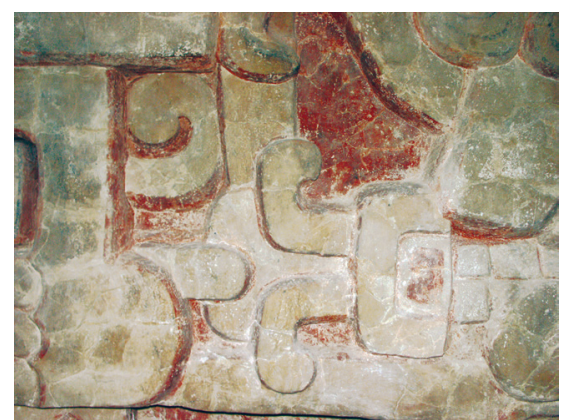

FIGURA 4. Modelo anatómico porcino, papel maché y escayola, depósito del Departamento de Anatomía y Anatomía Patológica Comparada (Anatomía y Embriología). Patrimonio Histórico Universidad Complutense de Madrid (Cortesía: Museo Veterinario Complutense [MVC], Universidad Complutense de Madrid [UCM]).

de los siglos XVI y XVII, en los palacios de la nobleza y la aristocracia europea aparecieron los llamados cuartos - o gabinetes - de maravillas, en los que se acopiaban y exhibían objetos raros o extraños, naturales o construidos por el hombre, llevados de muchas partes del mundo (Figura 5). Luego, al desarrollarse el movimiento cultural e intelectual de la Ilustración, entre finales del siglo XVII y principios del XIX, se crearon, tanto en Europa como en América, sociedades e instituciones que tenían como finalidad mejorar las disciplinas del conocimiento, en especial, las científicas; fue entonces cuando aquellos gabinetes, también Ilamados de curiosidades, dieron paso a los de ciencias, organizados metódicamente, como fueron los casos del muy célebre Gabinete Real de París, trabajado por el naturalista francés Georges-Louis Leclerc, conde de Buffon (1707-1788), o del Gabinete de Historia Natural de México, abierto hacia 1790 a expensas del también naturalista José Longinos Martínez, miembro de la Real Expedición Botánica a Nueva España, realizada entre 1787 y 1803 (Vega y Ortega 2011:3). Estos tipos superio- 
res de gabinetes son, justamente, los antecesores directos de los actuales museos veterinarios $y$, en definitiva, de los museos de arte, de ciencias y de historia natural.

Entre otros muchísimos museos veterinarios que hay en el mundo, vale señalar algunos que sobresalen por su trayectoria, organización y dimensión: el Museo de Historia Veterinaria de la facultad correspondiente de la Universidad Complutense (MHV-FV-UCM) (Figura 6); el Museo y Archivo del Cuerpo de Veterinaria Militar de España (Cemilvet), ambos en Madrid; el American Museum of Veterinary Medicine (AMVM), en Pennsylvania, Estados Unidos de América; el Musée de la Médecine Vétérinaire (MMV) de I'Université de Liège, en Bélgica; el Veterinærhistorisk Museum (VMH), de Copenhage, Dinamarca; el Veterinärmedizinhistorisches Museum (VMHM) de Tierärztlichen Hochschule, Hannover, Alemania, y el Muzeum Weterynarii przy Muzeum Rolnictwa, en Polonia.

La World Association for History of Veterinary Medicine (WAHVM) ha considerado que esta práctica museística es estratégica para la recuperación del patrimonio veterinario y el fortalecimiento de la identidad profesional; en este sentido, bajo su auspicio, promoción y patrocinio, se creó un grupo de trabajo integrado por los curadores de estos museos en más de 28 países, cuya tarea consiste en diseñar un futuro Programa de Intercambio de Materiales, así como editar la Guía de museos veterinarios, ambos en continuo proceso de formulación (WAHVM 2009). Los avances en estas responsabilidades se dan a conocer periódicamente y la información se actualiza cada dos años, en ocasión del Congreso Mundial de esta asociación (WAHVM 2012:2), el cual incluye, por cierto, una sesión académica especialmente dedicada a las actividades y la temática de los museos veterinarios (WAHVM 2009).

Venezuela no está aislada de esta realidad; en ella destaca el Sistema Museístico del Decanato de Ciencias Veterinarias de la Universidad Cen-

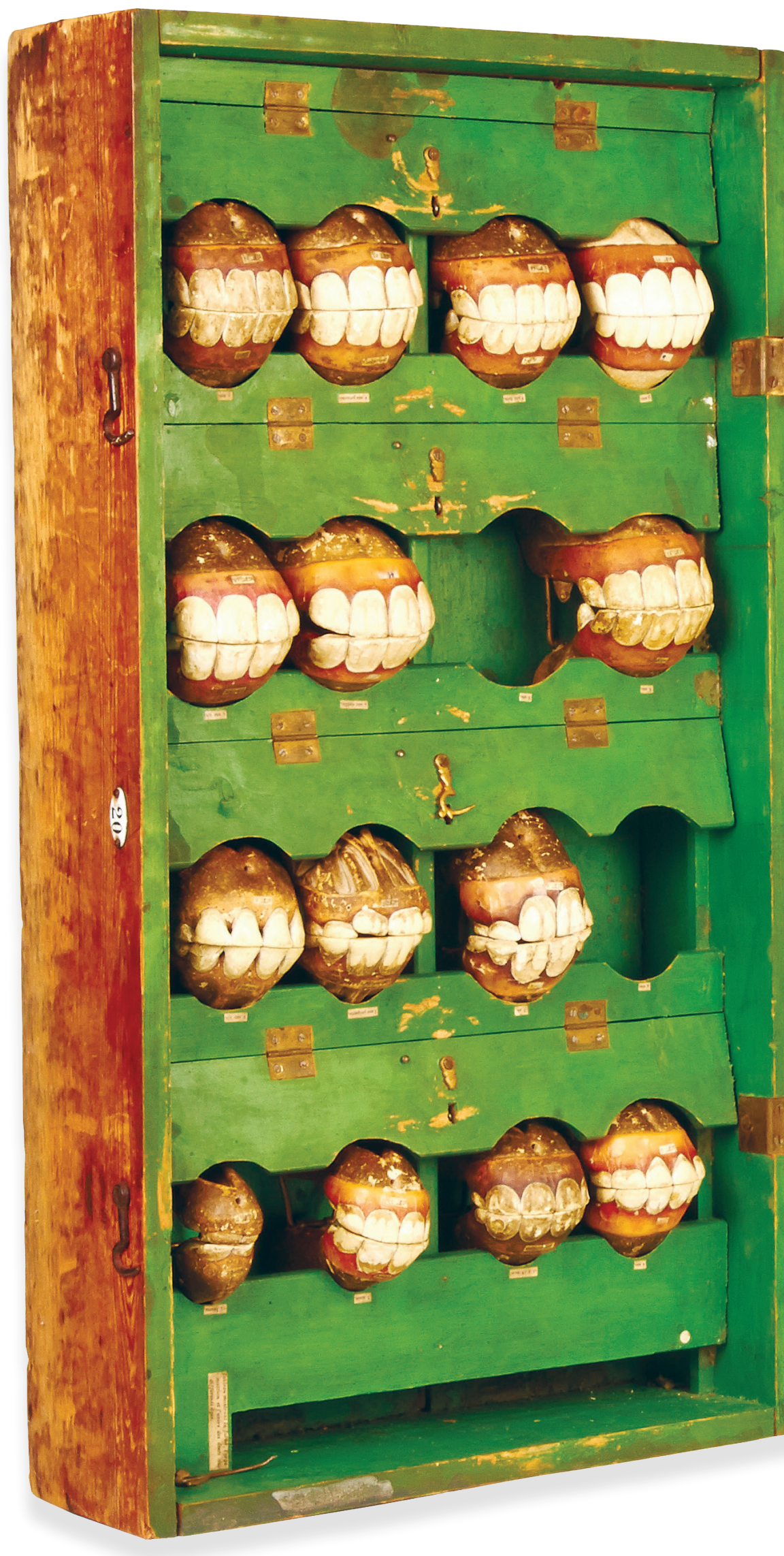




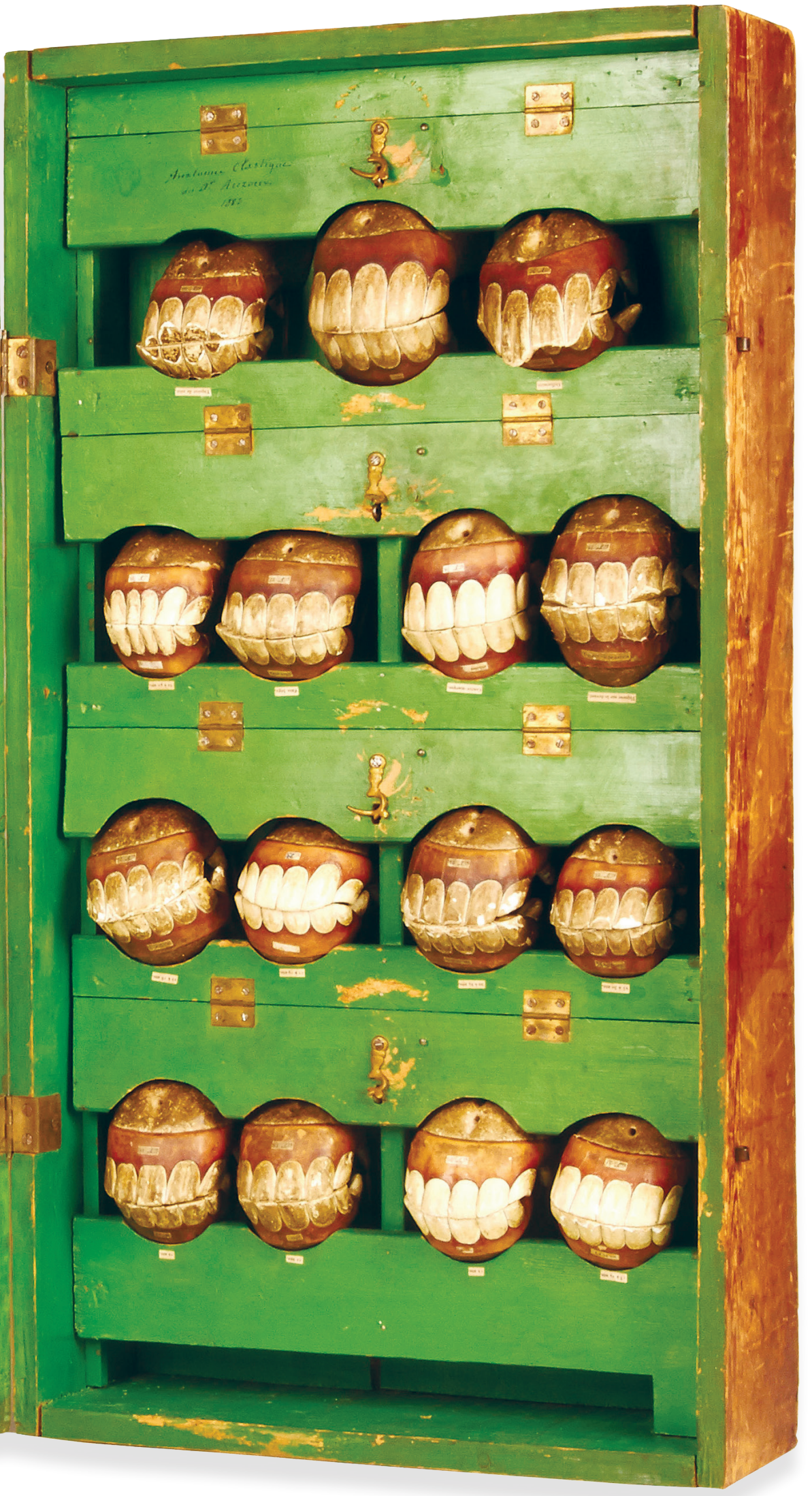

troccidental Lisandro Alvarado (DVCUCLA), en Barquisimeto, conformado por los museos de Anatomía y de Patología Animal (Figura 7), así como por la Vitrina Histórica y la Biblioteca de Historia Veterinaria Dr. José Hernández Romero; existen, por lo demás, iniciativas similares en cuatro de las seis facultades actualmente en funcionamiento en el país: en las universidades Central de Venezuela (UCV), del Zulia (LUZ) y las nacionales experimentales Francisco de Miranda (UNEFM) y Rómulo Gallegos (UNERG); en las dos restantes: las nóveles escuelas de las también universidades nacionales experimentales de Los Llanos Ezequiel Zamora (UNELLEZ) y Simón Rodríguez (UNESR) no existe, por su muy reciente creación, tal experiencia, pero de seguro pronto se asumirá su reto en este sentido, dada la importancia que reviste para la formación, la historia y la identidad médico veterinaria.

\section{Conclusiones}

Los sistemas museísticos veterinarios, además de colaborar en la preparación y la formación de identidad profesional de los futuros médicos veterinarios, cumplen la función social de sensibilizar al público en general en cuanto al conocimiento y la valorización de los animales, al mismo tiempo que contribuye a la construcción de la ciudadanía, al hacer aportaciones a la historia nacional desde la particular visión de la historia de la medicina veterinaria y la ganadería, así como de la cultura de mascotas.

Sin embargo del cumplimiento de tales propósitos, persiste un gran reto por afrontar: la optimización constante y con criterios técnicos de estos centros en cuanto a la adecuación y

FIGURA 5. Estuche de maxilares y mandíbulas de equino mostrando la evolución con el tiempo de la dentición y algunos defectos de la misma de Louis Thomas Jerôme Auzoux, papel maché, depósito del Departamento de Producción Animal. Patrimonio Histórico Universidad Complutense de Madrid (Cortesía: Museo Veterinario Complutense [MVC], Universidad Complutense de Madrid [UCM]). 

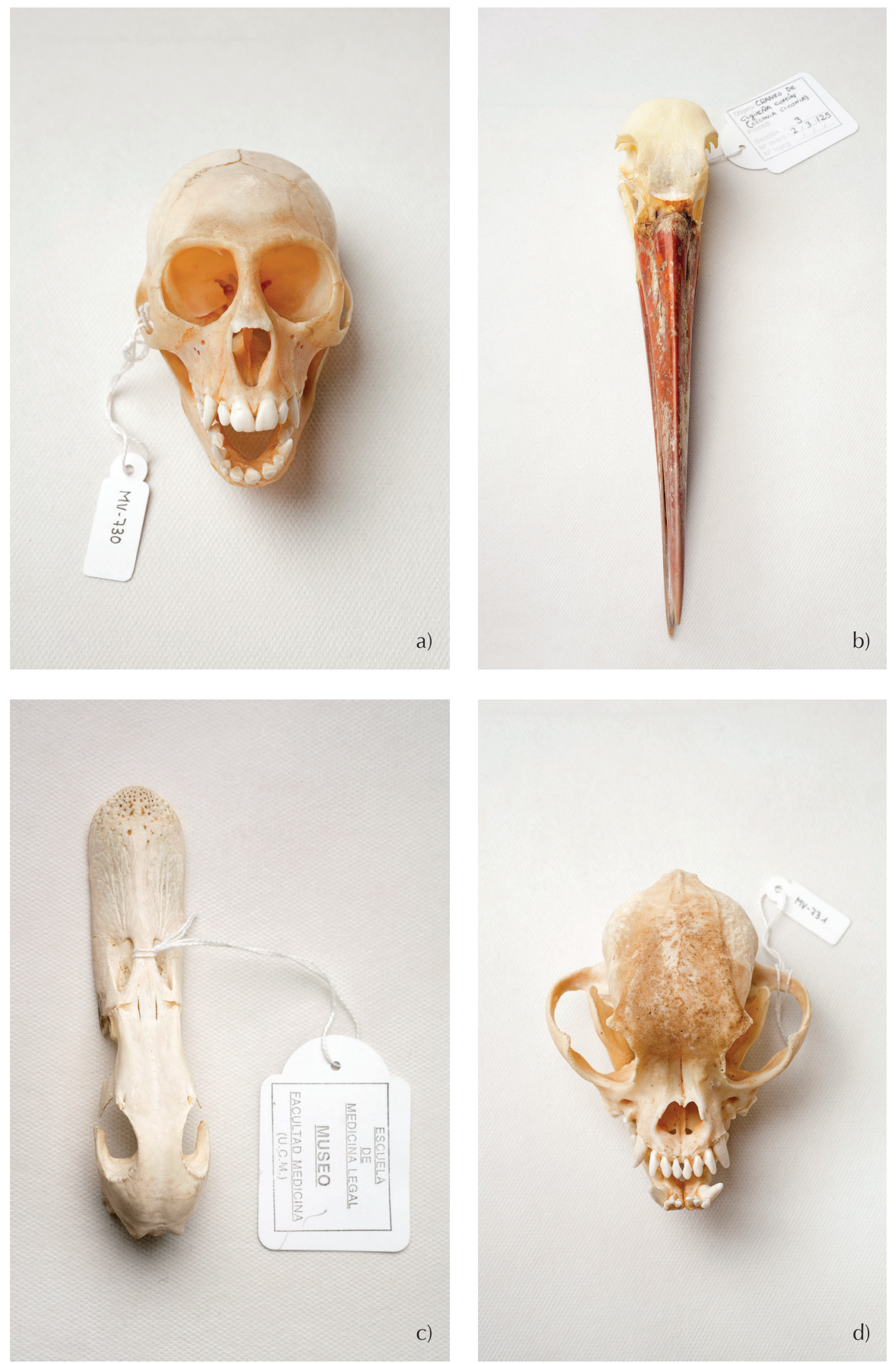

FIGURA 6. Cráneos de: a) primate, hueso, depósito del Museo de Antropología Forense (UCM); b) cráneo de cigüeña común (ciconia ciconia), hueso, donación Museo Reverte; c) cráneo de pato S.P., hueso, donación Museo Reverte y d) cráneo de perro (Caniche), hueso, depósito del Departamento de Anatomía y Anatomía Patológica Comparada (Anatomía y Embriología). Patrimonio Histórico Universidad Complutense de Madrid (Cortesía: Museo Veterinario Complutense [MUC], Universidad Complutense de Madrid [UCM]). 


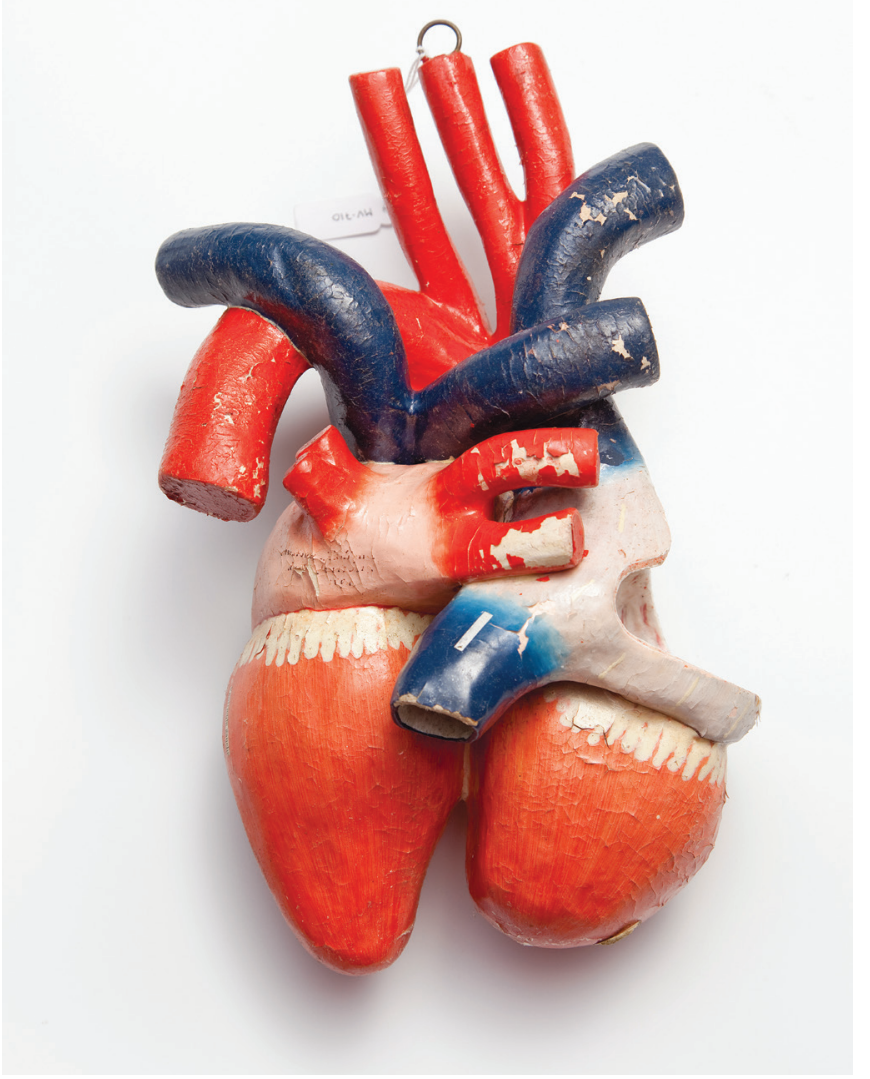

FIGURA 7. Corazón de Dugong de Louis Thomas Jerôme Auzoux, papel maché, depósito del Departamento de Anatomía y Anatomía Patológica Comparada (Anatomía y Embriología). Patrimonio Histórico Universidad Complutense de Madrid (Cortesía: Museo Veterinario Complutense [MUC], Universidad Complutense de Madrid [UCM]).

expansión de espacios, la preparación del personal tanto en museística como en conservación, el crecimiento y mejora de las colecciones, la posibilidad del autofinanciamiento por medio de la generación de ingresos por visitas o publicaciones, y muy primordialmente, la adhesión de defensores y colaboradores mediante un trabajo de divulgación para que la sociedad en general entienda la importancia que reviste, en la comprensión del presente $y$ el diseño del futuro, el resguardo del pasado.

\section{Referencias}

Ábalos Calvo, Cristina, Paula Cuenca Escalera y Noelle Rodríguez Garrido

2007 “El Museo de Veterinaria: la recuperación del patri- monio", Revista Complutense de Ciencias Veterinarias, Madrid, 1 (2):532-536.

CEIP Fernando Guanarteme de Gáldar

2012 “2. 3. Manifiesto Día del Museo-2008: La importancia de los museos en el desarrollo social", Carnaval de Lectura, Perú, 2012:8-9, documento electrónico disponible en [http://issuu.com/goethelima/docs/boletinmayo/8], consultado en febrero del 2016.

Degueurce, Christophe

2012 "Les collections historiques vétérinaires: un enjeu pour l'avenir?", Revista del Colegio Médico Veterinario del Estado Lara, 2 (4):16-22.

Sánchez de Lollano Prieto, Joaquín y Celia Rodríguez Varela 2009 "Nace el Museo Veterinario Complutense", Profesión Veterinaria, Revista del Colegio de Veterinarios de Madrid, 71:86-88.

Vega y Ortega, Rodrigo A.

2011 "La riqueza del Gabinete de Historia Natural del Museo Nacional de México. La década de 1830", Nuevo Mundo. Mundos Nuevos, documento electrónico disponible en [http://nuevomundo.revues.org/62082\#ftn1], consultado en mayo del 2016.

WAHVM

2009 Working Groups, documento electrónico disponible en [http://www.wahvm.umn.edu/WorkingGroups.html], consultado en enero del 2016.

2012 Join Meeting of the Board and the Liaison Committee Minutes, World Association for the History of Veterinary Medicine, documento electrónico disponible en [http:// wahvm.org/wp-content/uploads/2013/07/2012UtrechtBoa rdMinutes.pdf], consultado en enero del 2016.

\section{Síntesis Curricular del/los autor/es}

\section{Naudy Trujillo Mascia}

Universidad Centroccidental Lisandro Alvarado (UCLA), Venezuela naudytrujillo@ucla.edu.ve

Médico veterinario con magister scientiarum y doctorado en historia. Presidente de la Sociedad Venezolana de Historia de la Medicina Veterinaria. Coordinador de la Cátedra de Historia, Ética y Deontología de la Medicina Veterinaria adscrita al Departamento de Ciencias Sociales y Económicas del Decanato de Ciencias Veterinarias de la Universidad Centroccidental Lisandro Alvarado (DCV-UCLA, Venezuela). Docente e investigador en el área de la historia de la ganadería, de la medicina veterinaria y las instituciones de salud y sanidad animal, de la genealogía de familias ganaderas en el centroccidente de Venezuela, así como en ética y deontología médico-veterinaria y bioética.

Postulado/Submitted 02.03.16

Aceptado/Accepted 31.05.16

Publicado/Published 11.07.16 